\title{
Design of 32-bit 3-Stage Pipelined Processor based on MIPS in Verilog HDL and Implementation on FPGA Virtex7
}

\author{
Husainali S. Bhimani \\ Dept. of Electronics and \\ Communication \\ Charusat University, Changa
}

\author{
Hitesh N. Patel \\ Faculty of Electronics and \\ Communication \\ Charusat University, Changa
}

\author{
Abhishek A. Davda \\ Dept. of VLSI \& Embedded \\ Systems \\ DAIICT, Gandhinagar
}

\begin{abstract}
Reduced Instruction Set Compiler (RISC) is a microprocessor that had been designed to perform a small set of instructions, with the aim of increasing the overall speed of the processor. This paper presents 32 bit 3 stage architecture inspired by MIPS. The Idea of this paper is to implement custom architecture like MIPS 32 bit architecture in VERILOG HDL. The last step is to implement MIPS on FPGA (Field programmable gate array). MIPS (Microprocessor without Interlocked pipeline stages) processors are one of the first successful classical RISC architecture.
\end{abstract}

\section{Keywords}

MIPS, RISC, FPGA, VERILOG HDL.

\section{INTRODUCTION}

RISC stands for reduced instruction set computer. RISC processors are widely used processor nowadays. There are many advantages of RISC over CISC (complex instruction set computer) architecture. Those are high performance, fixed length instruction, single cycle execution, smaller size, and short development time. RISC design resulted in computers that execute instructions faster than other computers built of the same technology [5].Complexity is at software level rather than hardware level. One of the main advantages is pipelining which is absent in CISC processor. Pipelining improves overall performance of RISC architecture [2].

This paper presents architecture which is inspired by one of the very popular RISC architecture i.e., MIPS. MIPS stand for microprocessor without interlocked pipeline stages. It is an open source soft processor \& one of the first successful RISC architecture. MIPS are widely used in embedded systems. MIPS processor design is based on the RISC design principle that concentrates on load/store architecture [3]. MIPS has already been pronoun of MIPS instruction set and MIPS instruction set architecture [4].

This paper presents 3 stage RISC single cycle 32 bit pipelined processor inspired by MIPS having large set of registers. This architecture covers all basic instructions for general computation including load/store instructions.

\section{INSTRUCTION FORMAT}

Proposed architecture supports Two Types of instructions.

These are R-type instructions and J-type instructions. It considers two source registers, one destination register, shift value and opcodes for instructions.

\subsection{Type: 1 General instruction format}

\begin{tabular}{|c|c|c|c|c|}
\hline \multicolumn{5}{|c|}{ 32 Bit Instruction } \\
\hline $31-26$ & $25-19$ & $18-12$ & $11-05$ & $04-00$ \\
\hline Opcode & RS & RT & RD & SH \\
\hline
\end{tabular}

Fig 1: R-Type Instructions Format

Figure 1 shows R-Type Instructions Format.

5 bits: for shifting or rotating operation $\left(2^{\wedge} 5=32\right.$ bits).

6 bits: opcode $\left(2^{\wedge} 6=64\right)$ to support instructions up to 64 .

7 bits: To access registers up to $127^{\text {th }}$. (R0-R127)

Example:

1. ADD r0, r1, r2;

$\mathrm{r} 0=\mathrm{r} 1+\mathrm{r} 2$

2. AND r5, r6, r7;

$\mathrm{r} 5=\mathrm{r} 6 \& \mathrm{r} 7$

\subsection{Type: 2 Jump instruction format}

\begin{tabular}{|c|c|c|}
\hline \multicolumn{3}{|c|}{ JMP Instruction Format } \\
\hline $31-26$ & $25-07$ & $06-00$ \\
\hline Opcode & 000000000000000000000 & Target address \\
\hline
\end{tabular}

Fig 2: Jump type Instruction Format

Figure 2 shows Jump type Instruction Format

Example: JMP LABEL

Suppose LABEL is located at $125^{\text {th }}$ instruction then PC will immediately jumps to $125^{\text {th }}$ instruction.

\section{LIST OF INSTRUCTIONS AND OPCODE FOR REGISTERS}

\subsection{Instructions and their opcodes}

Table 1 shows list of instructions included in purposed architecture with respective opcodes. 
Table 1. Opcode for Instructions

OPCODE FOR INSTRUCTIONS

\begin{tabular}{|c|c|}
\hline OPCODE & INSTRUCTION \\
\hline 000000 & $\mathrm{ADD}$ \\
\hline 000001 & $\mathrm{ADC}$ \\
\hline 000010 & SUB \\
\hline 000011 & SBC \\
\hline 000100 & LSL \\
\hline 000101 & LSR \\
\hline 000110 & CMP \\
\hline 000111 & NOP \\
\hline 001000 & MOV \\
\hline 001001 & AND \\
\hline 001010 & OR \\
\hline 001011 & NOT \\
\hline 001100 & XOR \\
\hline 001101 & JMP \\
\hline 001110 & ROTL \\
\hline 001111 & ROTR \\
\hline 010000 & INC \\
\hline 010001 & DEC \\
\hline 010010 & ZERO \\
\hline 010011 & LOAD \\
\hline 010100 & STORE \\
\hline
\end{tabular}

\subsection{Registers and their Opcodes}

Table 2 shows how register is accessed with their opcode.

Registers can be accessed like R0 as 0000000 , R1 as 0000001 , $\mathrm{R} 2$ as $0000010, \mathrm{R} 3$ as $0000011, \mathrm{R} 4$ as $0000100, \mathrm{R} 5$ as 0000101, R6 as 0000110, R7 as 0000111, R8 as 0001000, and so on.

Table 2. Opcode for Registers

\begin{tabular}{|c|c|}
\hline Rs/Rt Rd & Accessed Register \\
\hline 0000000 & R0 \\
\hline 0000001 & R1 \\
\hline 0000010 & R2 \\
\hline 0000011 & R3 \\
\hline$\cdot$ & $\cdot$ \\
\hline$\cdot$ & $\cdot$ \\
\hline$\cdot$ & $\cdot$ \\
\hline 1111111 & $\cdot$ \\
\hline
\end{tabular}

\section{INSTRUCTION MEMORY, REGISTER FILE AND DATA MEMORY}

Here Instruction memory, register file and data memory are illustrated as blocks.

\subsection{Instruction memory}

Size: 128 bits

Figure 3 shows Instruction memory block.
Cache [127]

Cache [6]

Cache [5]

Cache [4]

Cache [3]

Cache [2]

Cache [1]

Cache $[0]$

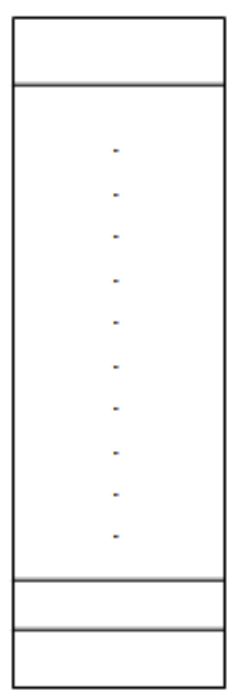

Instruction

Memory/Cache

Fig 3: Instruction memory

\subsection{Register File}

Figure 4 shows Register File block.

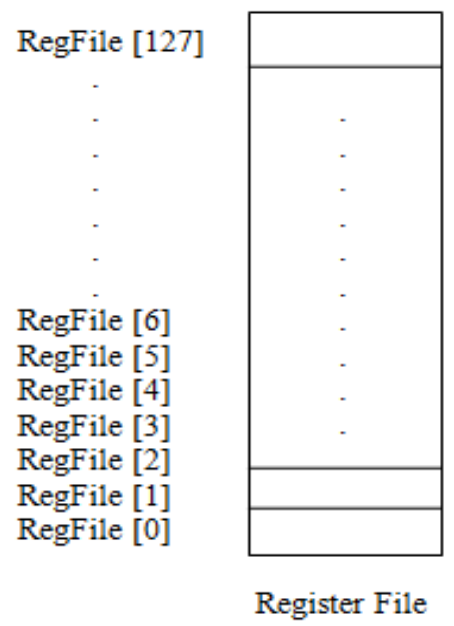

Fig 4: Register File

\section{FLAG STRUCTURE}

Figure 6 shows Flag structure.

Size of flag is 32 bits.

Flag [0]: Zero flag. It is updated on zero output of ALU.

Flag [1]: Carry flag. It is updated if there is carry/borrow generated by addition/subtraction operations.

Flag [2]: Parity flag. It is set for odd 1's in result of ALU. It will be cleared for even 1's in result of ALU.

Flag [3]: Overflow flag. It is updated as set if there is any overflow in result.

Flag [4] - Flag [31]: Future use. 


\begin{tabular}{|c|c|c|c|c|}
\hline \multicolumn{5}{|c|}{ FLAG } \\
\hline FLAG[31]-FLAG[4] & FLAG[3] & FLAG[2] & FLAG[1] & FLAG[0] \\
\hline Future Use & Over Flow Flag & Parity Flag & Carry Flag & Zero Flag \\
\hline
\end{tabular}

Fig 6: Flag structure

\section{ARCHITECTURE}

There are two architectures covered here. First one is original MIPS and second one is modified custom architecture inspired by MIPS.

\subsection{MIPS Architecture [1]}

Figure 7 shows classical MIPS architecture which is single cycle RISC architecture. It was designed by John L. Hennessy at Stanford University. It is first classical and successful RISC processor which is of 5 stages i.e. instruction fetch, instruction decode, instruction execute, memory access and write back. Instruction Fetch module fetches instruction. Next module decodes it. Execute module executes the instruction. Memory access is required for load and store instruction. Write back module writes data from ALU to register file or loads data from data memory. The width of data bus of MIPS is 32 bits.

\subsection{Proposed Architecture}

Figure 8 shows Fetch stage of proposed architecture.

Instruction fetch: This is the first step in which instructions are fetched from instruction memory according to the address provided by PC. After each clock PC will be incremented according to the previous instruction fetched. Pipe1 is of 64 bits size. It saves 32 bits for instruction and 32 bits as next PC.

\subsubsection{Decode Stage}

Figure 9 shows decode stage of proposed architecture.

Instruction decode: This stage decodes instructions, shift value, registers to be accessed $\&$ getting their content.

\subsubsection{Control Signals}

Table 3 shows control signals part-1 for processor.

Table 4 shows control signals part- 2 for processor.
Pipe 2 is of 124 bits size. It saves 14 bits of CSTSIG which is primarily used to control the whole processor.

Pipe2 [13:00]: CSTSIG [13:00].

Pipe2 [19:14]: Future use.

Pipe2 [52:20]: 32 bits Address into file register (generally). For store instruction it gives data.

Pipe2 [83:52]: Rtout.

Pipe2 [115:84]: Rsout.

Pipe2 [120:116]: shift/rotate use.

Pipe2 [115:84]: future use.

Note that this architecture is with all control signals. Control signals can be considered as the heart of processor. All the critical decisions are taken by control logic of a processor.

\subsubsection{Execute/Data access/Write back}

Figure 10 shows Execute/Data Access/Write back stage of purposed architecture.

Instructions are executed in this stage. Data memory is accessed for load/store instructions. If it's not load/store instruction then result of ALU output is stored in register and flags are updated. Figure shows different required CSTSIG value for data memory and multiplexers.

Pipe 2 is of 124 bits size. It saves 14 bits of CSTSIG which is primarily used to control whole processor.

Pipe2 [13:00]: CSTSIG [13:00].

Pipe2 [19:14]: Future use.

Pipe2 [52:20]: 32 bits Address into file register (generally). For store instruction it gives data.

Pipe2 [83:52]: Rtout.

Pipe2 [115:84]: Rsout.

Pipe2 [120:116]: shift/rotate use.

Pipe2 [115:84]: future use. 


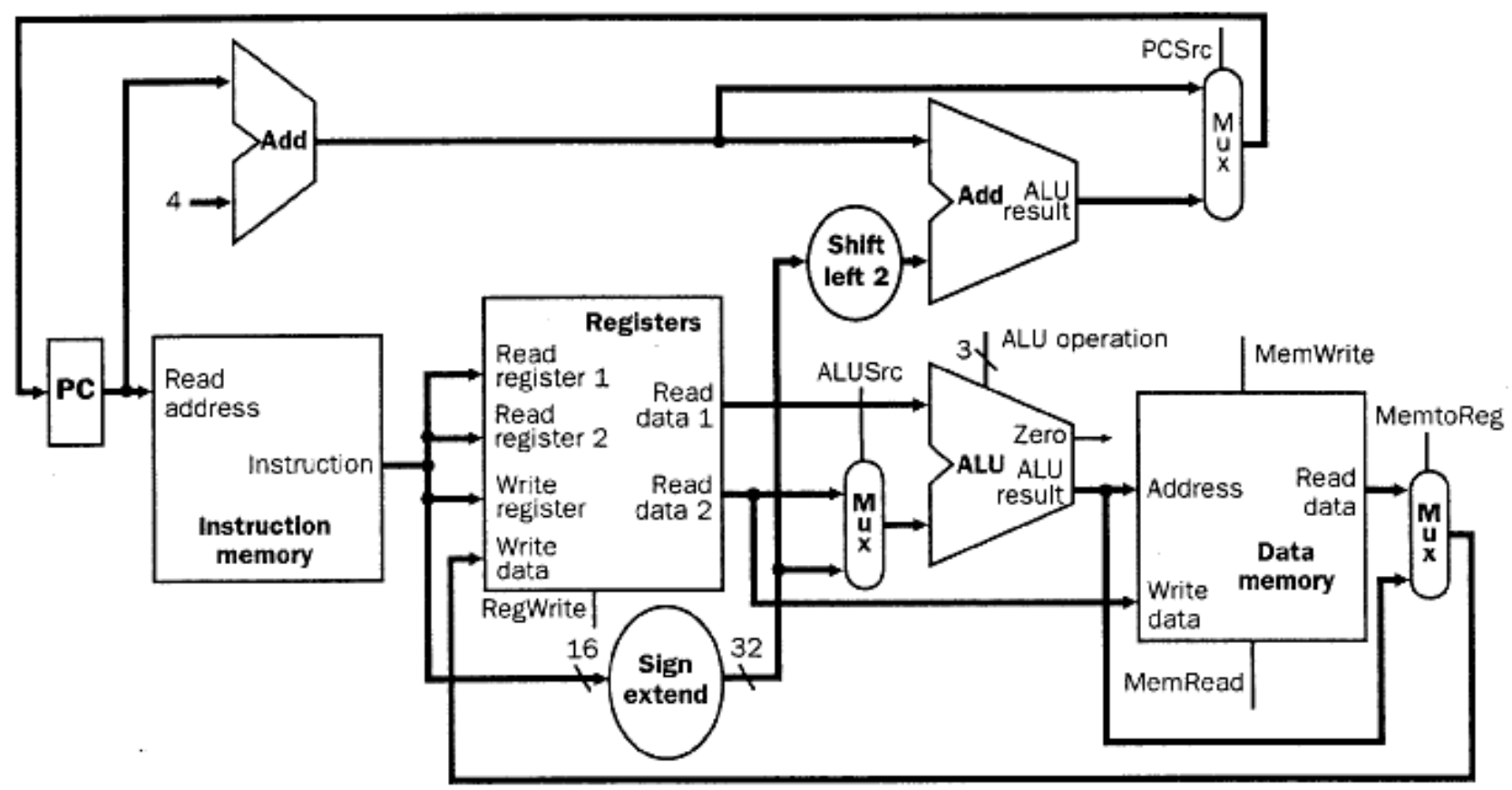

Fig 7: MIPS Architecture [1]

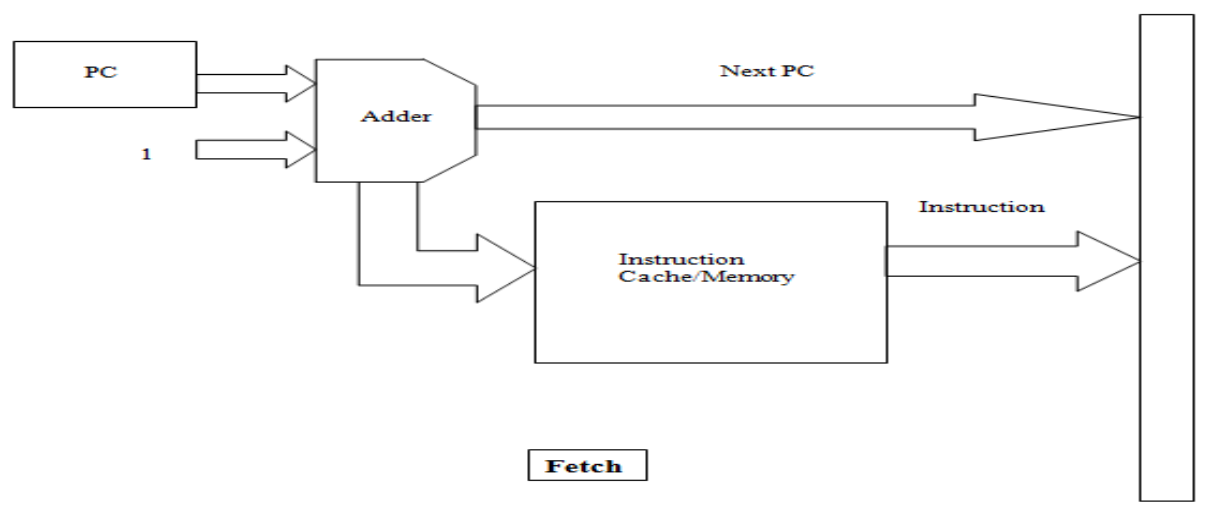

PIPE 1 [0:63]

$00: 31$ Next PC

32:63 Instruction

(32:36 Sh
$37=43$ Rdint

44:50 Rtint

51 57 Rsint
S8:63 Opcode)

Fig 8: Fetch Stage 


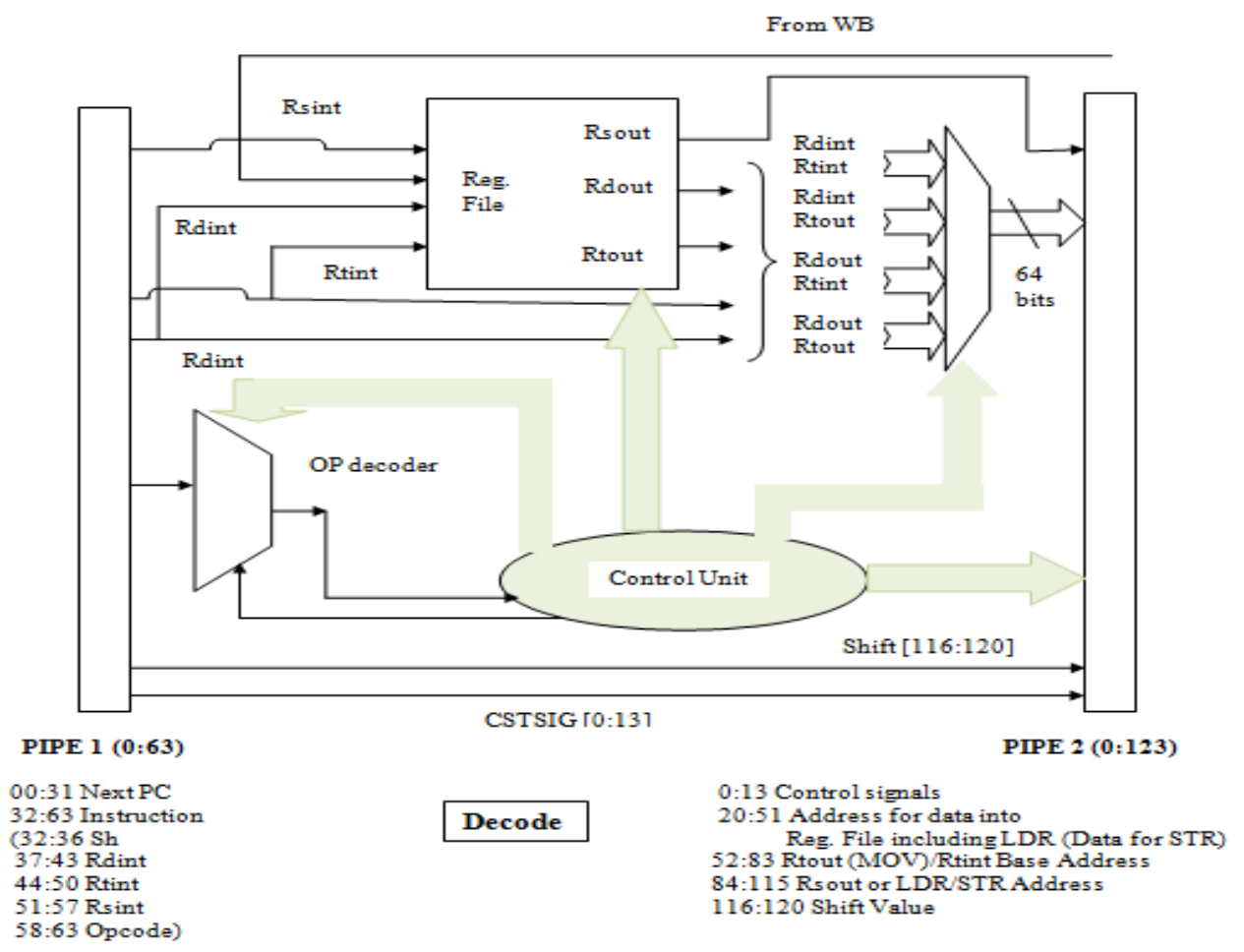

Fig 9: Decode Stage

Table 3. Control signals part-1

\begin{tabular}{|c|c|c|}
\cline { 2 - 3 } \multicolumn{2}{|c|}{ CSTSIG [0:13] } \\
\hline CSTSIG[n] & STATUS & DESCRIPTION \\
\hline CSTSIG[1] & 0 & Addition enabled including LDR,STR \\
& 1 & Subtraction enabled including CMP \\
\hline CSTSIG[8:7] & 00 & AND \\
& 01 & OR \\
& 10 & XOR \\
& 11 & NOT \\
\hline CSTSIG[0] & 0 & - \\
& 1 & Always asserted to update flags \\
\hline CSTSIG[10:09] & 00 & To enable INC \\
& 01 & To enable DEC \\
& 10 & To enable ZERO \\
& 11 & - \\
\hline
\end{tabular}


Table 4: Control signals part 2

\begin{tabular}{|c|l|c|}
\cline { 2 - 3 } \multicolumn{2}{c|}{ CSTSIG [0:13] } \\
\hline CSTSIG[n] & \multicolumn{1}{|c|}{ STATUS } & DESCRIPTION \\
\hline CSTSIG[4] & 0 & RdFinal address Disabled \\
& 1 & RdFinal address enabled \\
\hline CSTSIG[3] & 00 & LDR data from memory to register file \\
& 01 & ALU o/p or Rt (data to be moved) \\
\hline CSTSIG[2] & 0 & ALU o/p \\
& 1 & Rt (data to be moved) \\
\hline CSTSIG[12] & 0 & MemWrite Disabled \\
& 1 & MemWrite Enabled \\
\hline CSTSIG[11] & 0 & MemRead Disabled \\
& 1 & MemRead Enabled \\
\hline CSTSIG[1:0] & 00 & LSL \\
& 01 & LSR \\
& 10 & ROTL \\
& 11 & ROTR \\
\hline CSTSIG[13] & 0 & To enable ADC \\
& 1 & To enable SBC \\
\hline
\end{tabular}

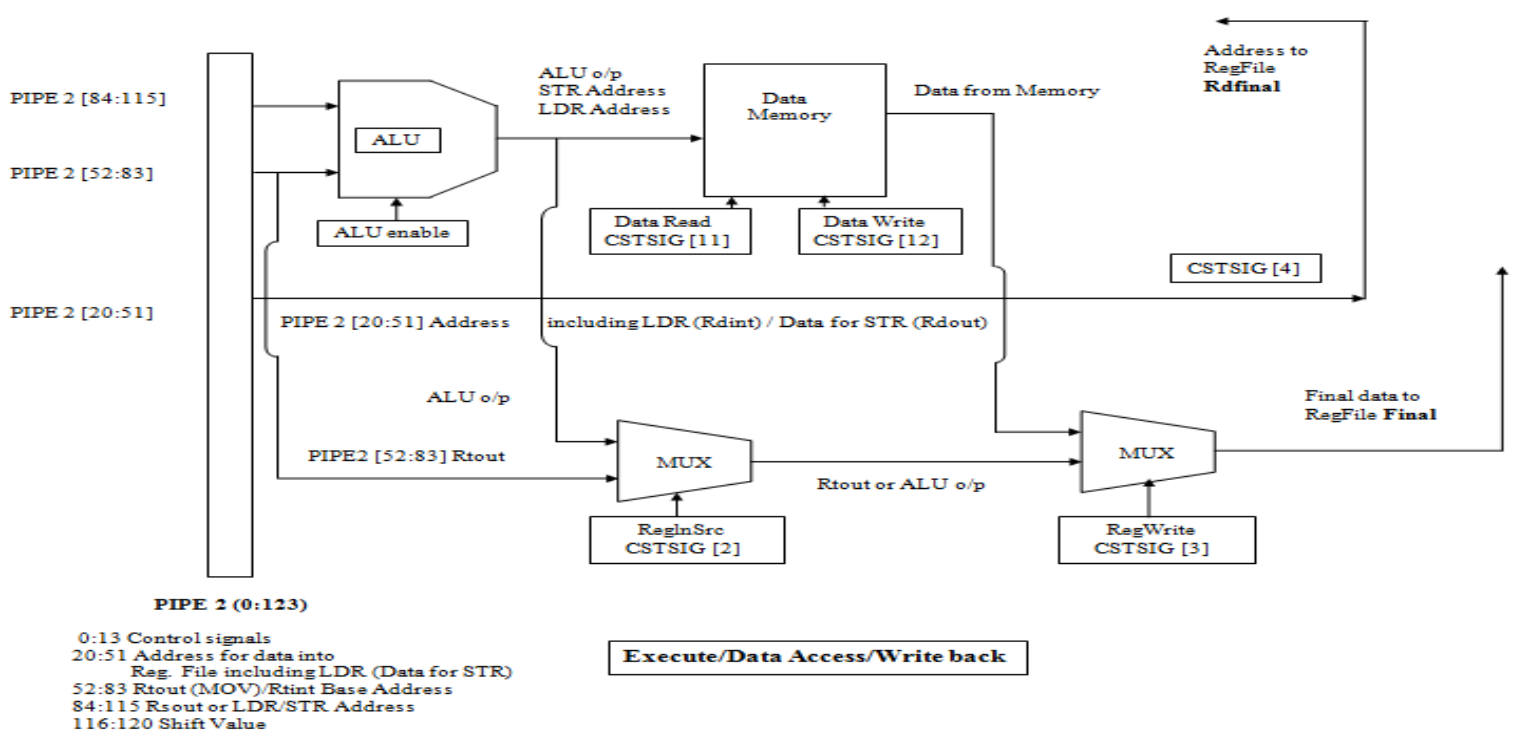

Fig 10: Execute/Data Access/Write back stage

\section{SIMULATION RESULTS}

ISIM is used for simulation and results have been verified. The complete project has been developed on Package: FFG1157, FPGA VIRTEX 7 and device is XC7VX330T. Different output patterns have been generated with RISC Instructions. Coding of processor is done in VERILOG HDL.

\subsection{RTL Schematic}

Figure 11 shows RTL Schematic of three stage processor.

\subsection{Technology Schematic}

Figure 12 shows Technology Schematic of three stage processor.

It shows different output values of Rdfinal (address into register file) and Final (data to be written into register file)

\subsection{Simulation Waveforms}

7.3.1 General simulation waveform Figure 13 shows General simulation waveform.

\subsection{Instruction Cache Simulation Waveform}

Figure 14 shows General simulation waveform.

7.5 Register File Simulation Waveform Figure 15 shows Register file simulation waveform

7.6 Data Memory Simulation Waveform Figure 16 shows Data memory simulation waveform. 


\subsection{Flag Simulation Waveform}

\subsubsection{Flag[0]- Flag[3]}

Figure 17 shows Flag[0]-Flag[3] simulation waveform.

\subsubsection{Flag[0]- Flag[31]}

Figure 18 shows Flag[0]-Flag[31] simulation waveform.

\subsection{XPower Analysis}

Figure 19 shows XPower Analysis of purposed architecture.

\section{DEVICE UTILIZATION SUMMARY}

Selected Device : 7vx330tffg1157-3

Table 5. Logic utilization

\begin{tabular}{|c|c|c|}
\hline Number of Slice Registers: & 32 out of 408000 & $0 \%$ \\
\hline Number of Slice LUTs: & 40 out of 204000 & $0 \%$ \\
\hline Number used as Logic: & 40 out of 204000 & $0 \%$ \\
\hline
\end{tabular}

Table 6. Logic distribution

\begin{tabular}{|c|c|c|}
\hline Number of LUT Flip Flop pairs used: & 62 & NA \\
\hline Number with an unused Flip Flop: & 30 out of 62 & $48 \%$ \\
\hline Number with an unused LUT: & 22 out of 62 & $35 \%$ \\
\hline Number of fully used LUT-FF pairs: & 10 out of 62 & $16 \%$ \\
\hline Number of unique control sets: & 4 & NA \\
\hline
\end{tabular}

Table 7. IO utilization

\begin{tabular}{|c|c|c|}
\hline Number of IOs: & 4 & NA \\
\hline Number of bonded IOBs: & 3 out of 600 & $0 \%$ \\
\hline
\end{tabular}

Table 8. Specific feature utilization

\begin{tabular}{|c|c|c|}
\hline Number of Block RAM/FIFO: & 1 out of 750 & $0 \%$ \\
\hline Number using Block RAM only: & 1 & NA \\
\hline $\begin{array}{c}\text { Number of } \\
\text { BUFG/BUFGCTRL/BUFHCEs: }\end{array}$ & 1 out of 200 & $0 \%$ \\
\hline
\end{tabular}

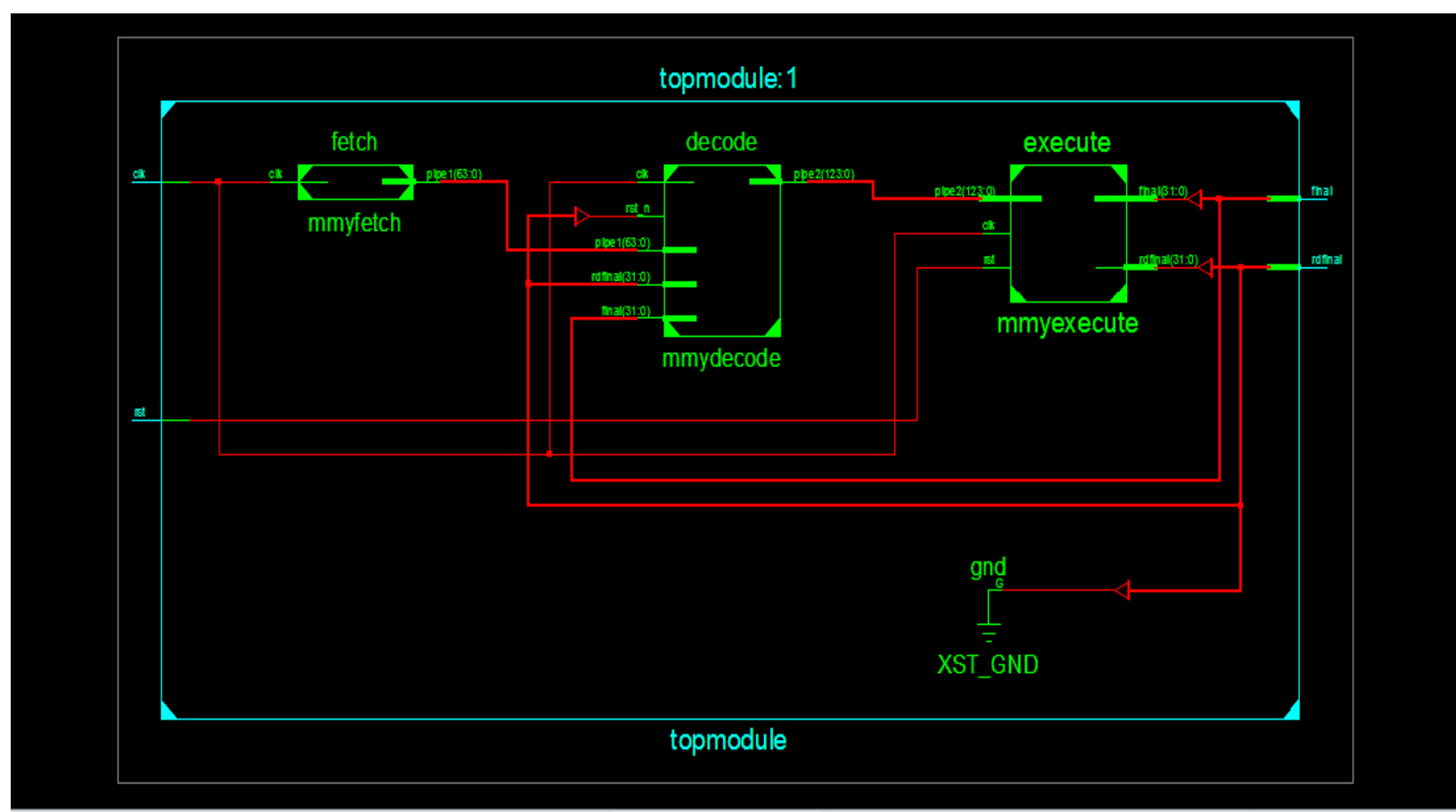

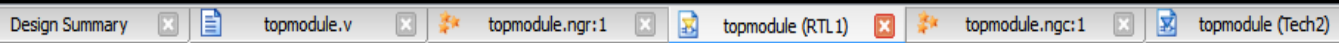

Fig 11: RTL Schematic 
International Journal of Applied Information Systems (IJAIS) - ISSN : 2249-0868

Foundation of Computer Science FCS, New York, USA

Volume 10-No.9, May 2016-www.ijais.org

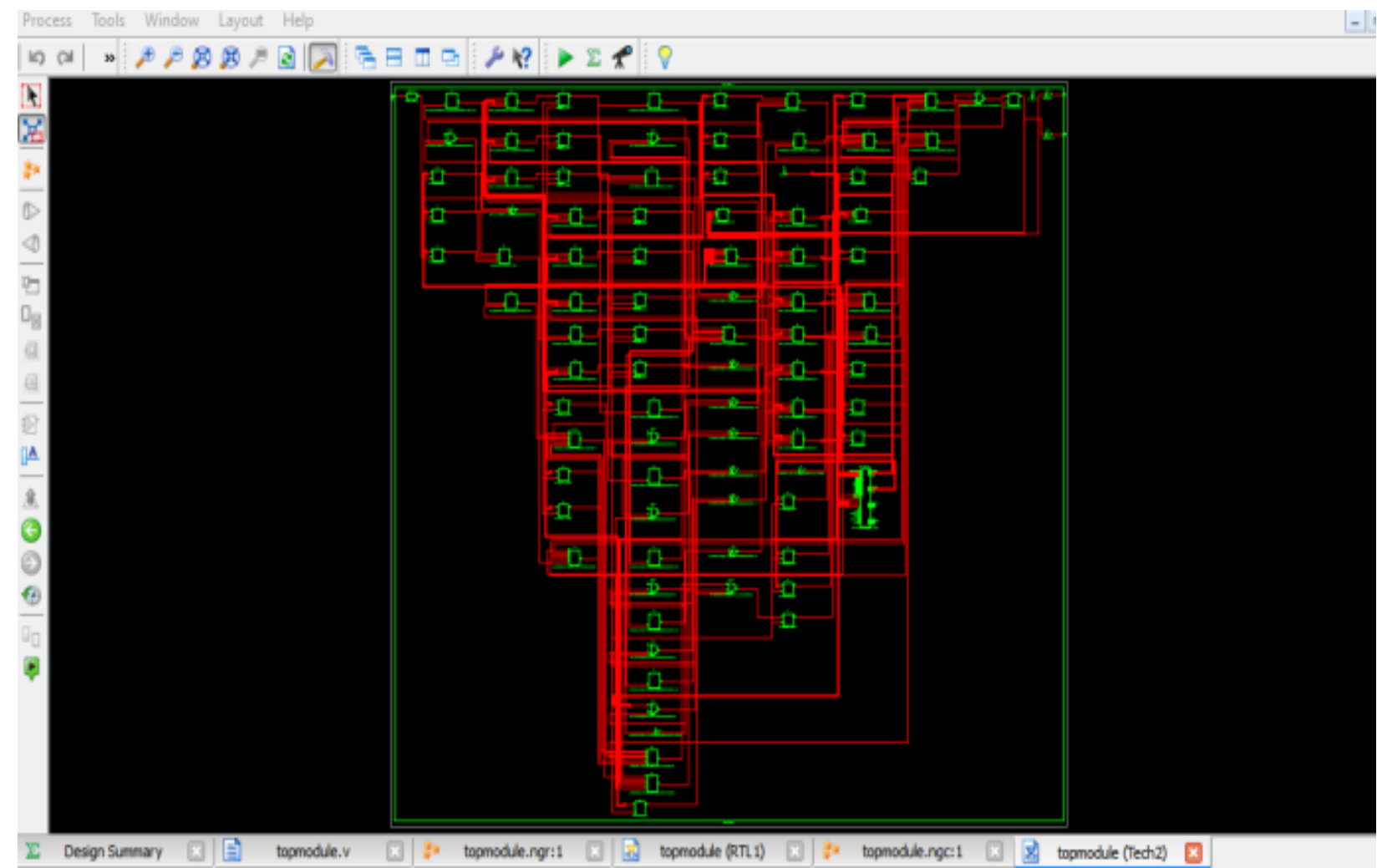

Fig 12: Technology Schematic

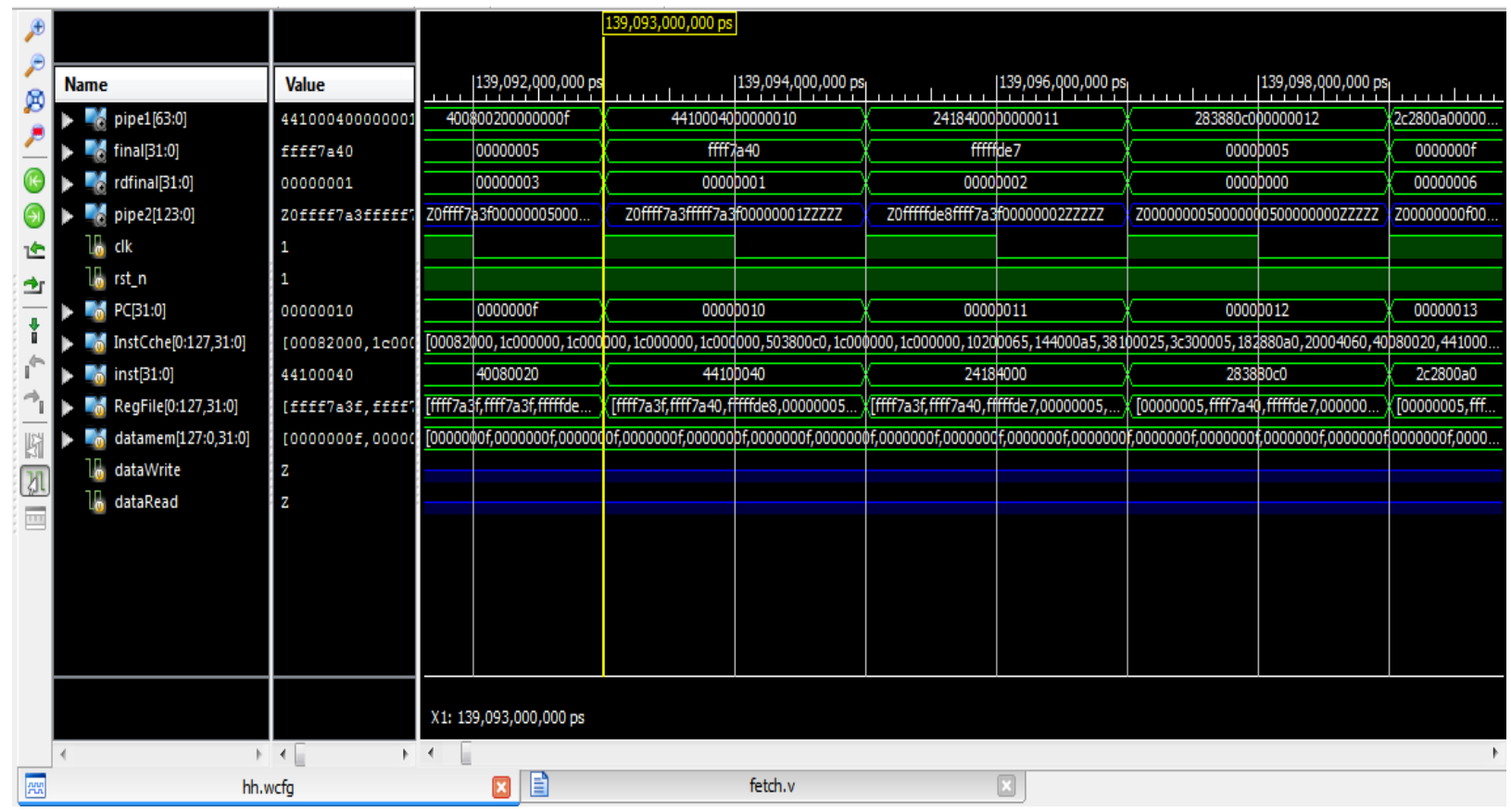

Fig 13: General simulation waveform 
International Journal of Applied Information Systems (IJAIS) - ISSN : 2249-0868

Foundation of Computer Science FCS, New York, USA

Volume 10-No.9, May 2016-www.ijais.org

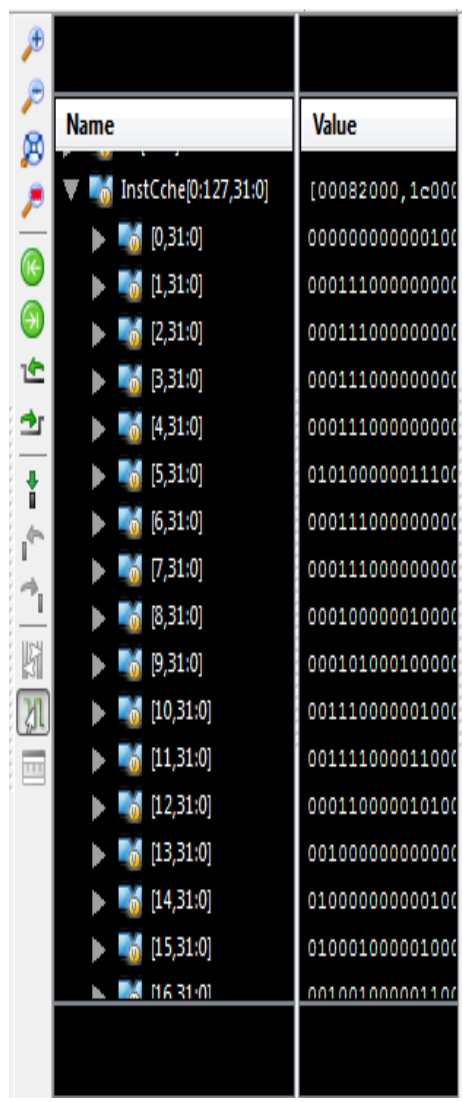

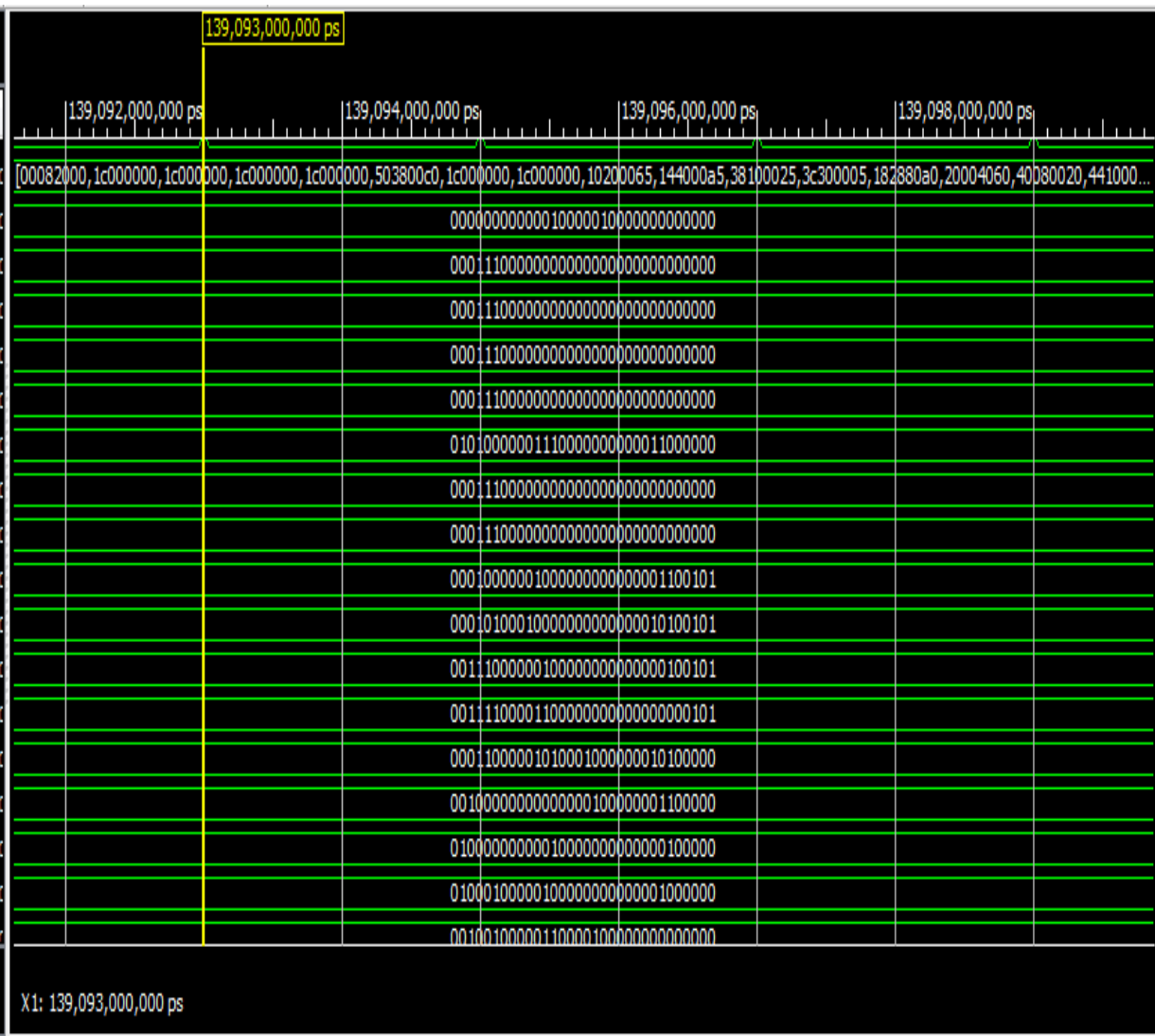

Fig 14: Instruction Cache Simulation Waveform

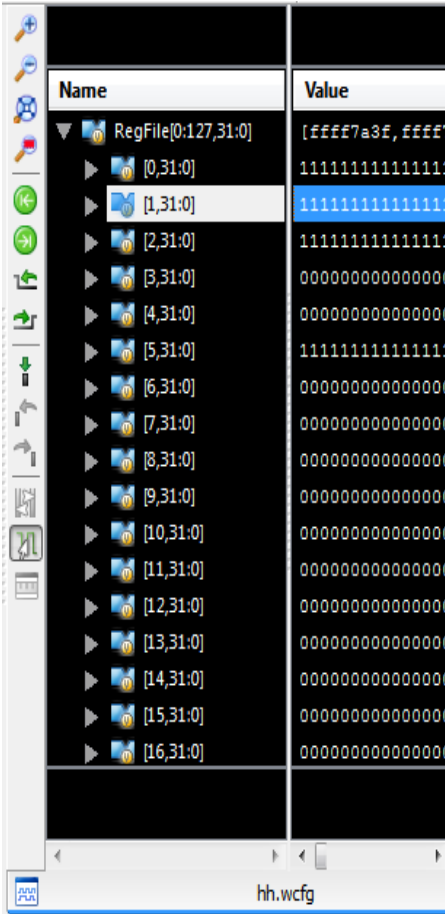

\begin{tabular}{|c|c|c|c|c|c|c|}
\hline & $139,093,000,000$ & $p s$ \\
\hline
\end{tabular}

Fig 15: Register file simulation waveform 
International Journal of Applied Information Systems (IJAIS) - ISSN : 2249-0868

Foundation of Computer Science FCS, New York, USA

Volume 10-No.9, May 2016-www.ijais.org

\begin{tabular}{|c|c|}
\hline Name & Value \\
\hline D $[15,31: 0]$ & 00000000000000 \\
\hline D $[14,31: 0]$ & 00000000000000 \\
\hline Dd $[13,31: 0]$ & 000000000000000 \\
\hline Dd $[12,31: 0]$ & 00000000000000 \\
\hline Dd $[11,31: 0]$ & 00000000000000 \\
\hline D $[10,31: 0]$ & 00000000000000 \\
\hline D $[9,31: 0]$ & 00000000000000 \\
\hline D $[8,31: 0]$ & 00000000000000 \\
\hline D $7,31: 0]$ & 00000000000000 \\
\hline D $[6,31: 0]$ & 00000000000000 \\
\hline D $[5,31: 0]$ & 00000000000000 \\
\hline D. $[4,31: 0]$ & 00000000000000 \\
\hline$D[3,31: 0]$ & 000000000000000 \\
\hline D $[2,31: 0]$ & 00000000000000 \\
\hline D $[1,31: 0]$ & 00000000000000 \\
\hline Do $[0,31: 0]$ & 00000000000000 \\
\hline 16 dataWrite & \\
\hline 10 dataRead & \\
\hline
\end{tabular}

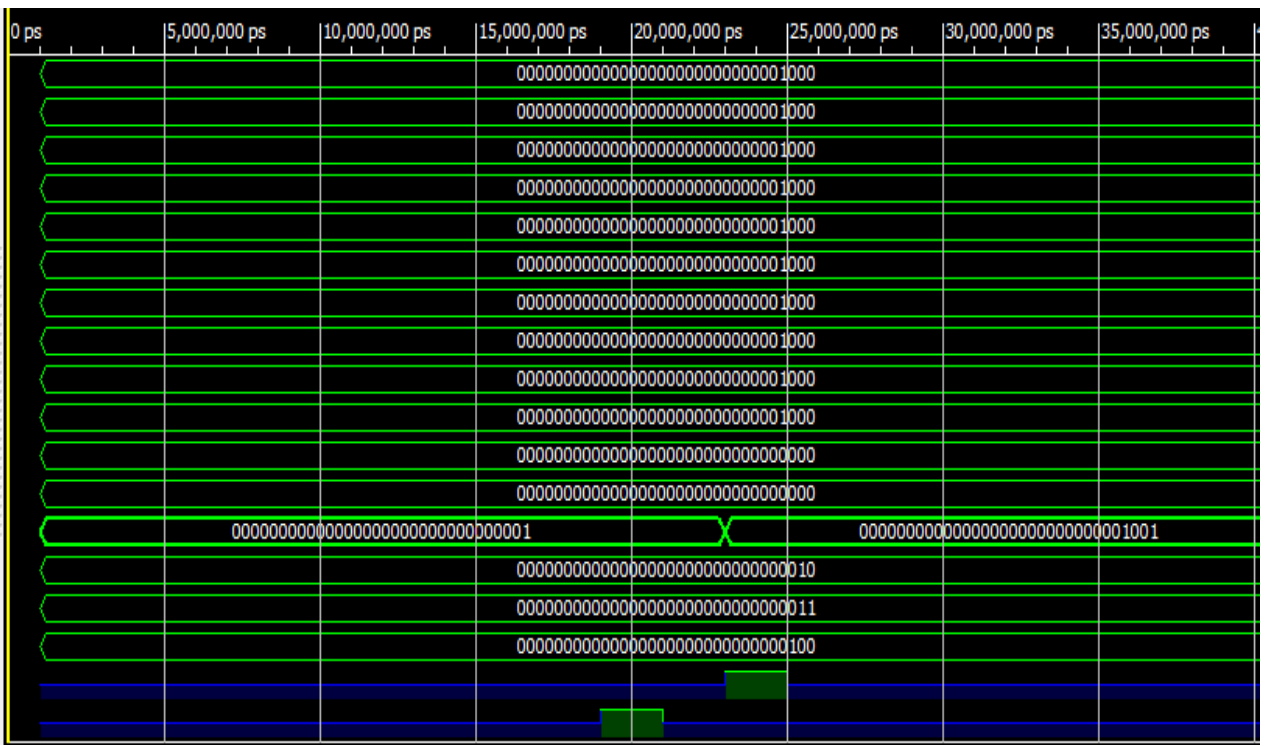

Fig 16: Data memory simulation waveform
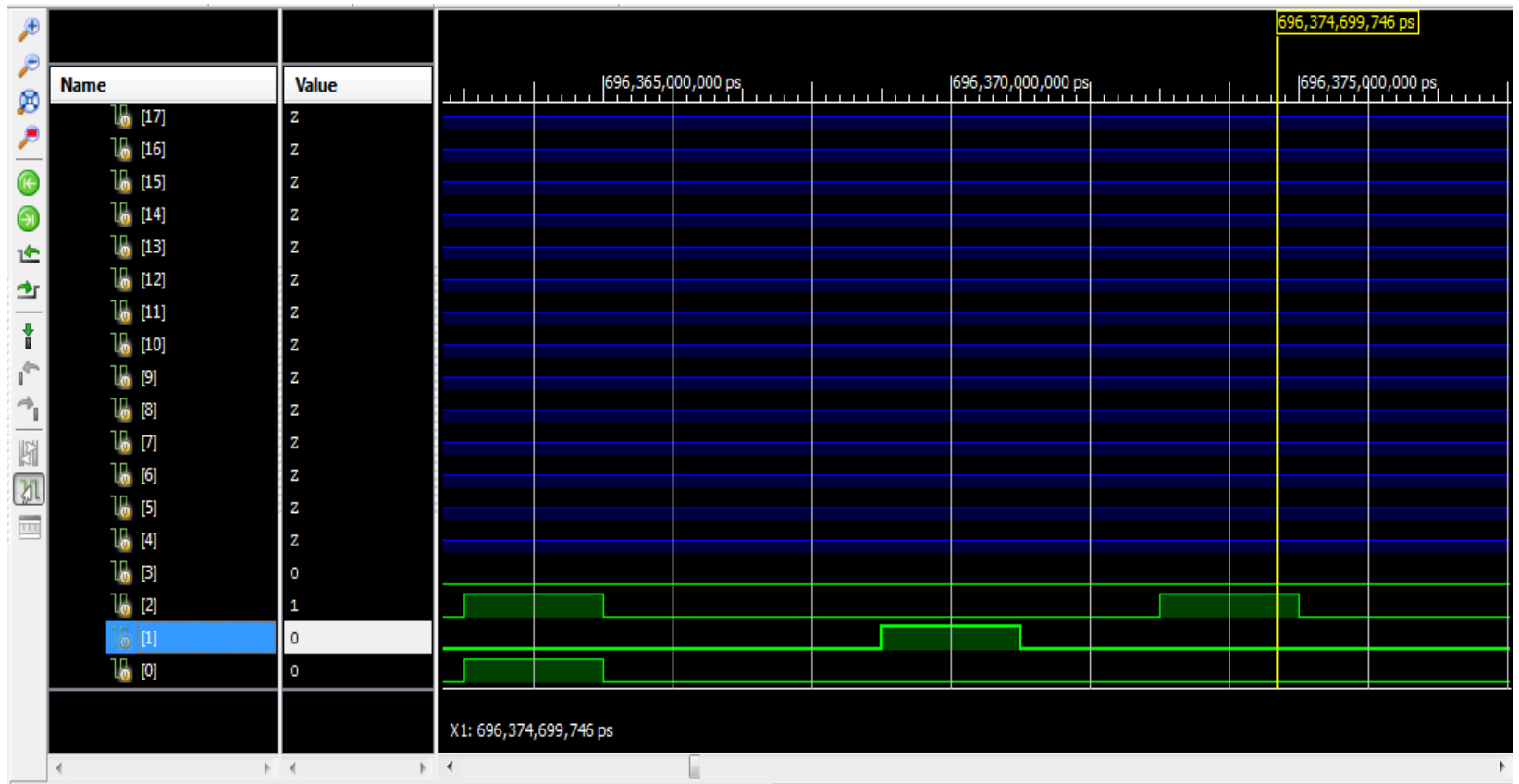

Default.wcfg*

$x$

Fig 17: Flag simulation waveform 


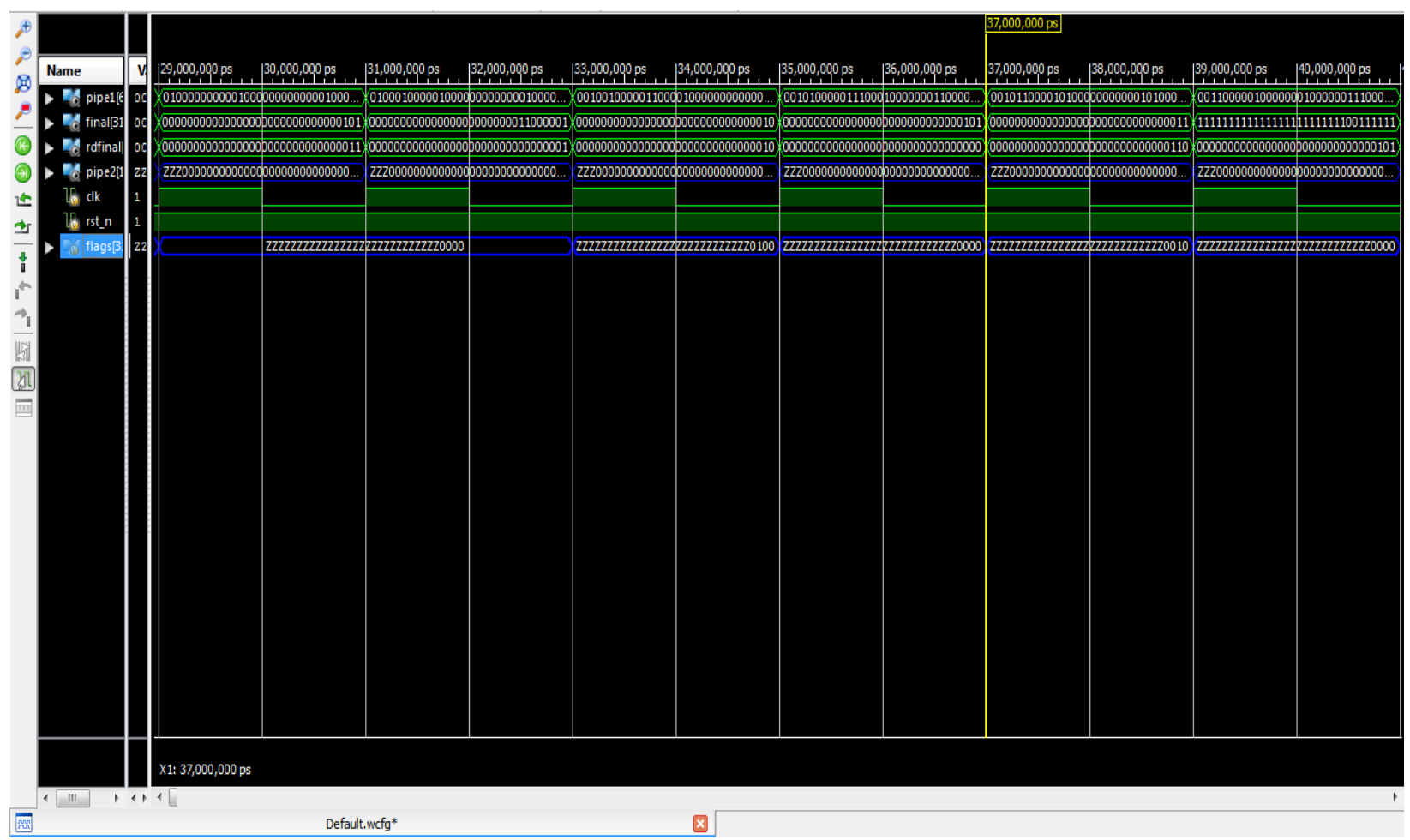

Fig 18: Flag simulation waveform

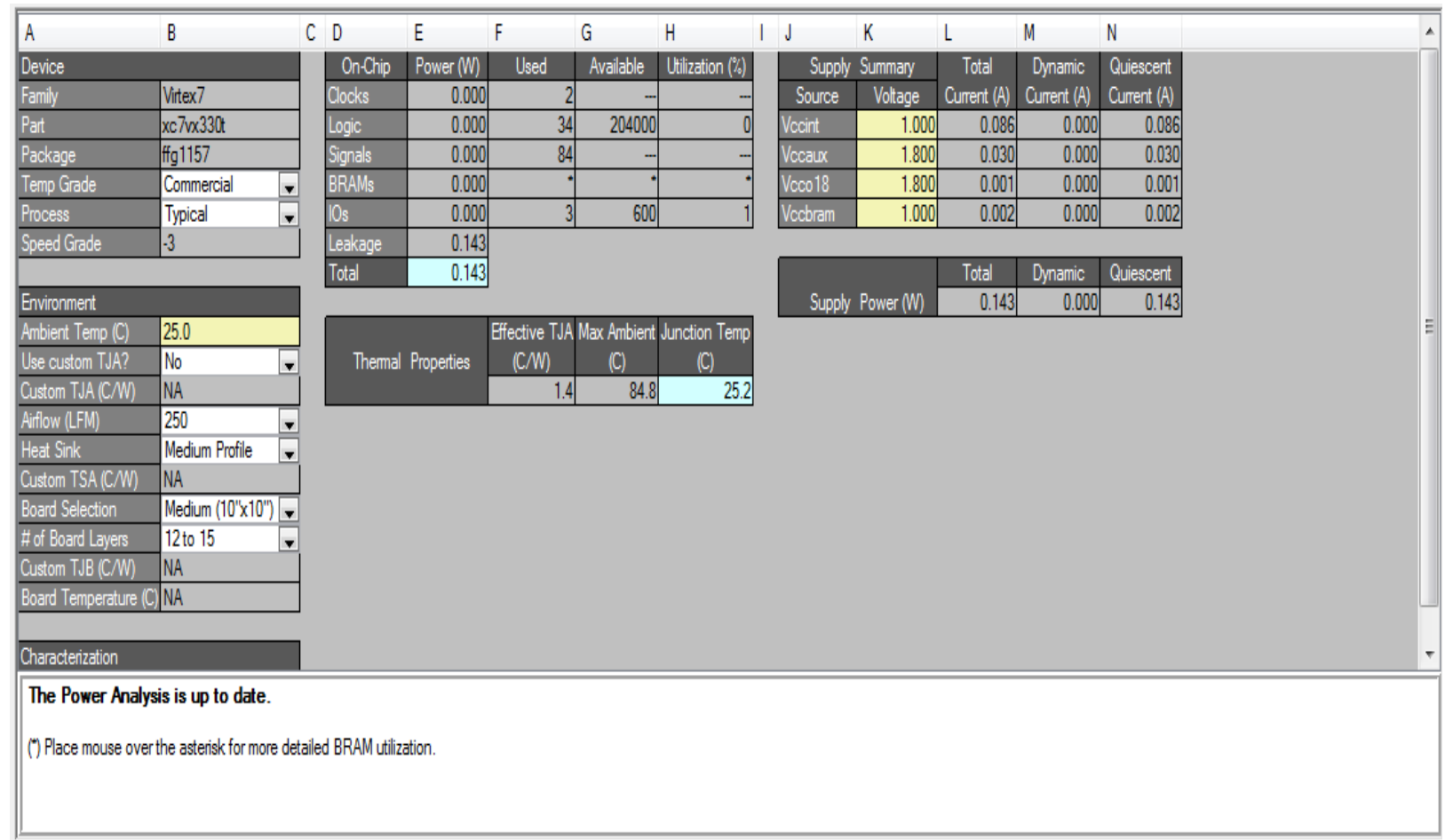

(5) Table View

Fig 19: XPower Analysis

\section{DESIGN SUMMARY}

$\begin{array}{lll}\# & \text { GND } & : 1 \\ \# & \text { INV } & : 2 \\ \# & \text { LUT1 } & : 7 \\ \# & \text { LUT2 } & : 3\end{array}$




$\begin{array}{lll}\# & \text { LUT3 } & : 11 \\ \# \quad \text { LUT4 } & : 7 \\ \# \quad \text { LUT5 } & : 4 \\ \# \quad \text { LUT6 } & : 6 \\ \# \quad \text { MUXCY } & : 7 \\ \# \quad \text { MUXF7 } & : 2 \\ \# \quad \text { VCC } & : 1 \\ \# \quad \text { XORCY } & : 8 \\ \# \quad \text { Flops/Latches } & : 32 \\ \# \quad \text { FD } & : 22 \\ \# \quad \text { FDR } & : 8 \\ \# \quad \text { LDE } & : 2 \\ \# \quad \text { RAMS } & : 1 \\ \# \quad \text { RAMB18E1 } & : 1 \\ \# \quad \text { Clock Buffers } & : 1 \\ \# \quad \text { BUFGP } & : 1 \\ \# \quad \text { IO Buffers } & : 2 \\ \# \quad \text { OBUF } & : 2 \\ \text { Coding: Verilog } & \\ \text { Implementation: } & \\ \text { Family: Virtex7 } & \\ \text { Device: XC7VX330T } & \\ \text { Package: FFG1157 } & \\ \text { Synthesis Tool: XST (VHDL/Verilog) } & \\ \text { Simulator: ISim (VHDL/Verilog) } \\ \text { \# }\end{array}$

\section{FEATURES}

Covers all basic instructions for general computation

3 stage processor

32 bit processor

Single cycle Processor

Pipelining Implemented for faster operation

Based on MIPS

Frequency: $310.878 \mathrm{MHz}$

Clock Period: $3.217 \mathrm{~ns}$
Data Memory: 128 bits

Instruction Memory Size: 128 bits

Register Files: 128 registers

Large Register File (R0-R127)

Supports load store instructions.

Supports arithmetic, logical and comparison operation

Supports full rotation and shifting of 32 bits.

No structural hazard since separate data memory and instruction memory is used.

\section{CONCLUSION}

This work successfully proposes a custom design of 3 stage pipelined RISC processor architecture. Pipeline is implemented and every instruction is tested using test bench. Proposed architecture supports large set of registers (R0R127). Design is simulated and synthesized using Xilinx ISE. Static timing analysis and power analysis of the design has been carried out. Feature such as data forwarding unit can also be added in future. The design can be synthesized using Cadence RTL complier in future. Also complete ASIC flow till RTL to GDS II can be done using Cadence SOC Encounter and it is possible to analyze the complete physical design flow.

\section{ACKNOWLEDGEMENT}

We wish to express our heartfelt appreciation to our friends, families and many who have rendered their support for the successful completion of the paper, both explicitly and implicitly.

\section{REFERENCES}

[1] David A. Patterson, John L. Hennessy 2005. Computer organization and design, 3rd Edition, Elsevier.

[2] Sharda P. Katke, G.P. Jain,"Design and Implementation of 5 Stages Pipelined Architecture in 32 Bit RISC Processor", IJETAE, Volume 2. Issue 4. April 2012, pp. 340-346.

[3] Preetam Bhosle, Hari Krishna Moorthy, "FPGA Implementation of low power pipelined 32-bit RISC Processor", International Journal of Innovative Technology and Exploring Engineering (IJITEE), August 2012.

[4] Bai-ZhongYing, Computer Organization, Science Press, 2000.11 .

[5] Charles E. Gimarc, Veljko M. Mhtinovic, "RISC Principles, Architecture, and Design", Computer Science Press Inc., 1989. 